\title{
Determination of Nectar Nicotine Concentration in N. attenuata
}

Eva Rothe, Matthias Schöttner, Danny Kessler and Ian T. Baldwin*

Department of Molecular Ecology, Max Planck Institute for Chemical Ecology, Jena, Germany *For correspondence: $\underline{\text { baldwin@ice.mpg.de }}$

[Abstract] In this protocol, the determination of the nicotine concentration in nectar of Nicotiana attenuata is described. This method is applicable for the investigation of small amounts of nectar (above $1 \mu \mathrm{l}$ ). It is a high-throughput protocol optimized and streamlined for one skilled person to process approximately 100 nectar samples per day.

\section{Materials and Reagents}

1. Nicotiana attenuata

2. Nicotine-D3 (Cambridge Isotope laboratories, catalog number: DLM-1818-0.5)

3. Ammonium hydroxide $25 \%$ (Fluka, catalog number: 44273-100 ML-F)

4. Methanol (Merck KGaA, catalog number: 1.06007.2500)

5. Solvent A (see Recipes)

6. Solvent B (see Recipes)

\section{Equipment}

1. Varian 1,200 triple quad LC-MS system

2. Phenomenex Gemini NX 50 × 2 mm column, $3 \mu \mathrm{m} \mathrm{C18} \mathrm{(Phenomenex,} \mathrm{catalog} \mathrm{number:}$ 593381-9)

3. $1.5 \mathrm{ml}$ Eppendorf tubes

4. $1.5 \mathrm{ml} \mathrm{GC}$ vials (www.wicom.de)

5. $25 \mu \mathrm{l}$ glass capillary (BLAUBRAND ${ }^{\circledR}$, intraMark) (BRAND GMBH + CO KG, Wertheim, catalog number: 708722)

\section{Procedure}

1. The best time to collect nectar from $N$. attenuata is in the morning between 4:00 and 7:00 am, as the maximum nectar accumulation is reached around 4:00 am and remains stable until the sun rises or the lights in the glasshouse are switched on. As flowers of $N$. attenuate remain open for two nights, one must make sure that the same floral stage is 
used for collections. Usually newly opened flowers are used, which requires removal of all open flowers $24 \mathrm{~h}$ before the actual sampling in order to be sure to collect floral nectar from the same floral stage-- one- and two-day-old flowers are hard to tell apart from each other.

2. Nectar from flowers is collected by inserting a clean $25 \mu \mathrm{l}$ glass capillary into the corolla tube until it reaches the base of the nectaries (Figure 1). With practice, a complete nectar sample can be obtained by holding the capillary with one hand and the corolla tube with the other and removing the tube against the counter-pressure of the inserted capillary (Video 1). This technique requires some training in order to avoid damage to the ovary and contamination of the nectar. Alternatively, the complete corolla can be detached from the rest of the flower by simply pulling on the corolla tube. The nectar remains at the base of the tube from which it can be collected with a $25 \mu$ glass capillary (Video 2).

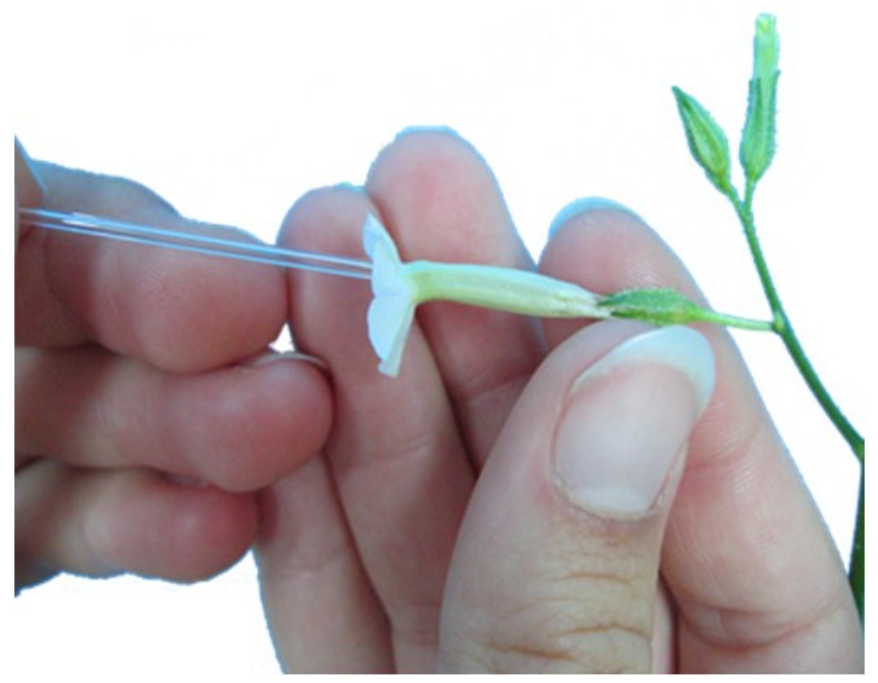

Figure 1. Floral nectar collection with a glass capillary

Video 1. Nectar collection from $N$. attenuata flowers - method 1

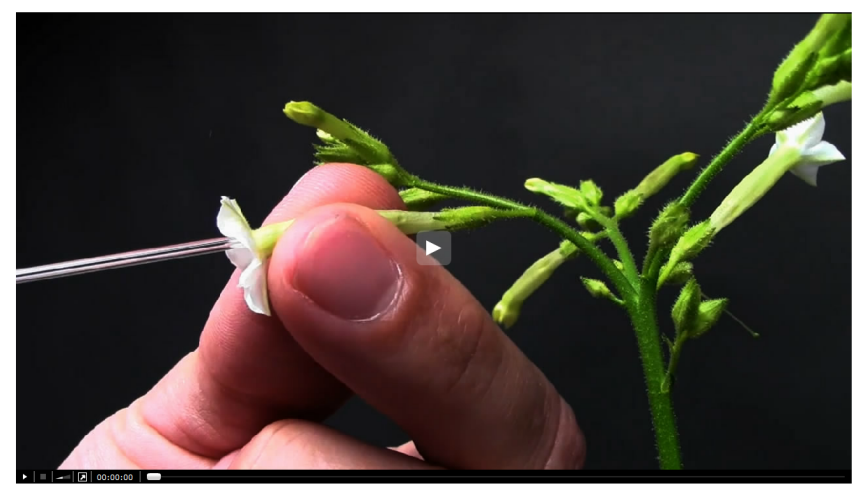


Video 2. Nectar collection from $N$. attenuata flowers - method 2

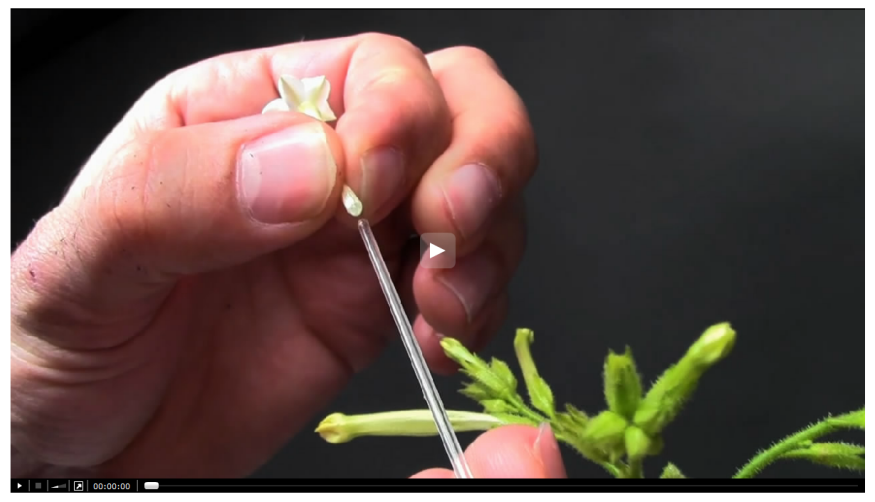

3. Nectar of single flowers is collected separately in $1.5 \mathrm{ml}$ Eppendorf tubes. $2 \mu \mathrm{l}$ or $1.5 \mu \mathrm{l}$ (for flowers with low nectar volume) of nectar are transferred into a new tube containing $400 \mu \mathrm{l}$ water and $20 \mathrm{ng}$ of the internal standard nicotine-D3.

4. Particles are removed by centrifugation ( $10 \mathrm{~min}$ at $12,000 \times \mathrm{g}$, at $4{ }^{\circ} \mathrm{C}$ ) and the supernatant is transferred into $1.5 \mathrm{ml} \mathrm{GC}$ vials.

5. $10 \mu \mathrm{l}$ of the solution are analyzed using a Varian 1,200 triple quad LC-MS system (http://www.varianinc.com) connected to an ESI source with a capillary voltage of $35 \mathrm{~V}$, solvent A ; Solvent B. The gradient (min/\% B): 0/5; 0.5/5; 2/80; 6.5/80; 8.5/5; 10/5 (Figure 2 ) is used with a Phenomenex Gemini NX $5 \times 2 \mathrm{~mm}$ column, particle size $3 \mu \mathrm{m}$. The $\mathrm{pH}$ of the mobile phase is the most important parameter which determines nicotine's retention on the column. The starting conditions focus nicotine on the column, while sugars and salts are eluted. The steep gradient guarantees sharp nicotine peaks. The time for reconditioning the column is strongly instrument dependent. The short reconditioning time in our program is adapted to accommodate our slow auto sampler. The transition of the precursor ion nicotine $[\mathrm{M}+\mathrm{H}]^{+}=163$ and nicotine-D3 $[\mathrm{M}+\mathrm{H}]^{+}=166$ to the fragment $(\mathrm{m} / \mathrm{z})=130$ at a collision energy of $14.5 \mathrm{~V}$ is recorded for quantification. Quantification was achieved by isotope dilution and can be calculated by the following formula: amount nicotine $(\mathrm{ng} / \mu \mathrm{l}$ nectar) = area of targeted compound/area ISD (internal standard $=$ nicotine - D3) $x$ amount ISD $(\mathrm{ng} / \mu \mathrm{l}$ nectar $)$. 


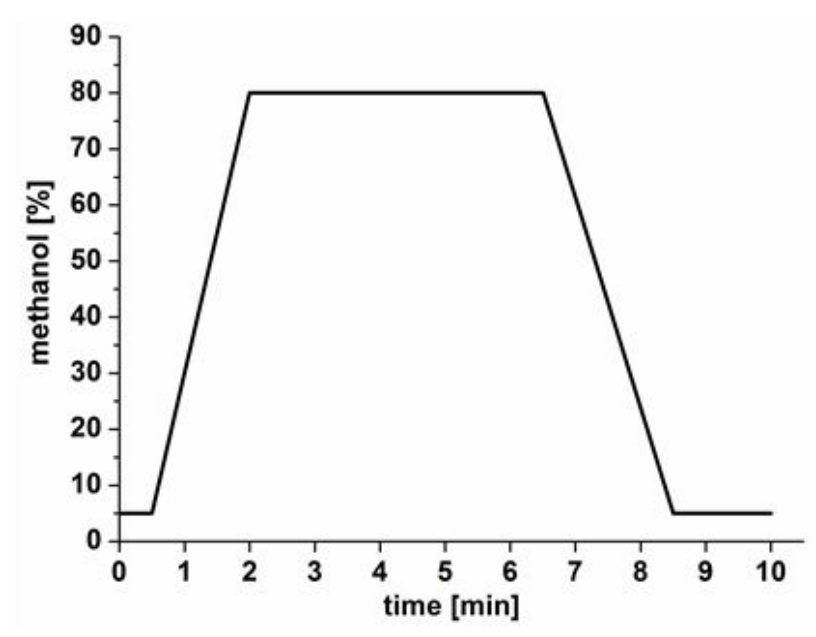

Figure 2. Methanol gradient

\section{$\underline{\text { Recipes }}$}

1. Solvent $A$

2. Add $1 \mathrm{ml}$ of $25 \%$ ammonium hydroxide solution to $1 \mathrm{~L}$ Milipore $\mathrm{H}_{2} \mathrm{O}$ Mix carefully, adjust pH to 10 [according to the pka of nicotine, which is 8.05 (Fujita et al., 1971) the $\mathrm{pH}$ should be 10 or higher, to maintain nicotine in its neutral form. The upper $\mathrm{pH}$ is limited by the column chemistry and may be adjusted with concentrated ammonia or a few drops of 1:10 diluted formic acid].

3. Solvent B

Methanol

\section{Acknowledgments}

This work was supported by the Max Planck Gesellschaft. The protocol was adapted from the publication: Kessler et al. (2012).

\section{References}

1. Fujita, T., Nakajima, M., Soeda, Y. and Yamamoto, I. (1971). Physicochemical properties of biological interest and structure of nicotine and its related compounds. Pesticide Biochem Physiol 1(2): 151-162.

2. Kessler, D., Bhattacharya, S., Diezel, C., Rothe, E., Gase, K., Schottner, M. and Baldwin, I. T. (2012). Unpredictability of nectar nicotine promotes outcrossing by hummingbirds in Nicotiana attenuata. Plant J 71(4): 529-538. 